\title{
I. Einführung
}

\author{
Franz-Xaver Kaufmann
}

\section{Diskurse über Staatsaufgaben}

\section{Zusammenfassung:}

Die vorherrschenden Auffassungen darüber, was Gegenstand staatlicher Geltungsmacht sein soll, haben sich im Zuge der neuzeitlichen Staatsentwicklung mehrfach geändert. Sie werden hier zu vier Diskursen - Polizeistaat, Rechtsstaat, Sozialstaat, Steuerungsstaat verdichtet, welche im Sinne eines Sequenzmodells auf die fortschreitende strukturelle Verselbständigung und funktionale Spezialisierung von gesellschaftlichen Teilsphären bezogen werden. Jüngste Umthematisierungen von Staatsaufgaben, wie sie auch den Gegenstand zahlreicher Beiträge dieses Bandes bilden, muten dem Staat nicht mehr problembezogen intervenierendes, sondern steuerndes, systembeeinflussendes Handeln zu. Dies hat nachhaltige Konsequenzen für die Staatstheorie und Staatspraxis: An die Stelle obsoleter Souverănitätsvorstellungen und dezidierter Hoheitsansprüche müssen lernfähige Formen der politischen Willensbildung und ein reflektierter Umgang mit dem Recht als dem Organisationsmittel des Staates treten.

Alle menschliche Ordnung ist symbolisch vermittelt. Das heißt, Menschen orientieren ihre Erwartungen, ihre wechselseitigen Beziehungen und ihr Handeln an Vorstellungen, welche nur insoweit mitteilbar und verständlich sind, aber auch überhaupt nur eine gewisse Festigkeit erhalten können, als sie Bezug nehmen auf sinnlich wahrnehmbare, bedeutungsvolle Signale, deren Bedeutung kulturell stabilisiert ist. Sprache ist dafür eine notwendige, aber keine hinreichende Bedingung. Nur insoweit als die Sprache mit ausreichender Verläßlichkeit auf erfahrbare Zusammenhänge verweist und deren Bedeutung stabilisiert, kann von Ordnung die Rede sein. "Sowohl nach ihrer Genese (Gesellschaftsordnung ist das Resultat vergangenen menschlichen Tuns) als auch in ihrer Präsenz in jedem Augenblick (sie besteht nur solange menschliche Aktivität nicht davon abläßt, sie zu produzieren) ist Gesellschaftsordnung als solche ein Produkt des Menschen* (Berger/ Luckmann 1969: 54). Kultur- und Gesellschaftsentwicklung als Vorstellung eines gerichteten (wenngleich von keinem Menschen so beabsichtigten) Prozesses läßt sich aufgrund unseres heutigen soziologischen Verständnisses imoderner، Gesellschaften als wachsende Komplexität der symbolischen Verweisungssysteme, als 
Ausdehnung der Rãume interdependenter sozialer Beziehungen und als zunehmende Differenzierung und Spezialisierung von Handlungssystemen begreifen. In diesem ProzeB kommt der Entstehung und Entwicklung des modemen Staates eine Schlüsselrolle zu. Der Staat ist selbst eine bestimmte Ordnungsvorstellung. und er gilt gleichzeitig als Garant gesellschaftlicher Ordnung. Die Vorstellung vom Staat ist jedoch nicht unwandelbar, seine Deutung ist nach Ort und Zeit ver. schieden, und ebenso variieren die von ihm ausgehenden Wirkungen. Diskurse. welche Bedeutung und Wirkung aufeinander beziehen, bedienen sich im deutschen Sprachraum häufig der Begriffe, Staatszwecker, 'Staatsfunktionen, oder 'Staatsaufgaben.. Wir können vier Stufen der Staatlichkeit oder Phasen der Staatsentwicklung unterscheiden, die sich durch die programmatische Dominanz unterschiedlicher Staatsaufgaben charakterisieren lassen. Sie sollen im Zusammenhang mit der erwähnten Vorstellung von Kultur- und Gesellschaftsentwicklung inter-
pretiert werden.

\section{Staatsaufgaben als staatstheoretisches Problem}

Der moderne Staatsbegriff unterscheidet sich von anderen Vorstellungen politisch verfaßter Ordnung vor allem durch den Gedanken der Einheitlichkeit seines Geund die römische Reichsordnung von ähnlichen Einheitsvorstellungen geleitet des römischen Reiches mit seiner festen atseinheit... das nie vergessene Vorbild Konzentrierung der Staatsgewalt" (Jellinek Organisation und Zentralisation, mit aber historisch durchaus wirksame Idinek 1966/1900: 317) eine retrospektive, mittelalterliche Sozialordnung, soweit sie politis darstellt. Auf jeden Fall war die solcher Einheitlichkeit weit entfernt sie politisch und rechtlich verfaßt war, von stets prekär und zumeist recht kurn. Zum einen blieben größere Reichsbildungen der seinem Ursprung noch seinem Inhalte existenz von Volksrecht und Herrscherrecht, ein einheitliches Recht. Die KoRecht, dieses wiederum sich ausdifferrecht, von kirchlichem und säkularem grundherrschaftlichen, kaufmännischen unierend in die Formen des feudalen, len Verhältnisse des Mittelalters (vgl. Berman 1983).

unzusammenhängenden Sozialordnung geprägten und im übrigen räumlich relativ Vorstellung des modernen Staates. Sie ist gekennzeich erst sehr allmählich die durch die Einheit des Territoriums einschließlich seineret in räumlicher Hinsicht in sozialer Hinsicht durch die Einheit des Herrschaftsverbanohner (Staatsvolk'), Hinsicht durch die Einheitlichkeit der Rechschaftsverbandes und in kultureller Elemente der Staatsvorstellung wurden im histordnung. Diese drei gedanklichen lung zunehmend aufeinander wurden im historichen Prozeß der StaatsentwickNationalstaaten des 19. Jahroezen, doch gelang es erst den konstitutionellen 
lung weitgehend zu realisieren. Im ihnen vorangehenden absolutistischen Staatswesen war es dagegen nicht gelungen, die fortdauernden regionalen und lokalen Rechte zu vereinheitlichen (vgl. Oestreich 1969).

Der Staat als historischer Realtypus einer durch einheitliches Recht und einheitliche Verwaltung konstituierten Gebietskörperschaft konnte sich somit erst als Verfassungsstaat realisieren, d.h. als eine sich begrenzende und durch Gewaltenteilung selbst kontrollierende politische Einheit, welche auf die Regelung aller sozialen Verhältnisse grundsätzlich verzichtete (Jellinek 1966/1900: 326). Während also in der absolutistischen Staatsvorstellung die Einheit des Staates gleichzeitig als Einheit von Staat und Gesellschaft gedacht wurde, konnte sich die staatliche Einheit historisch nur in dem Maße durchsetzen, als die zustande gekommene politische Einheitsordnung nicht mehr als das Ganze gesetzt, sondern als in sich begrenzte Einheit verstanden wurde, welche mit anderen, oft als igesellschaftlich, bezeichneten Ordnungen koexistierte. Erst in dem Maße, als die Ordnungsvorstellung des einheitlichen Staates vom Gedanken einer umfassenden Ordnung getrennt wurde, vermochte er sich historisch durchzusetzen. Daß der europäische Absolutismus nicht zur Despotie wurde, resultierte nicht nur aus der beschränkten Macht der Fürsten, sondern die Beschränkung dieser Macht war selbst eine Konsequenz der konkurrierenden Legitimationen und Rechtsordnungen. Die Einheitlichkeit der staatlichen Ordnung mußte daher mit der Freigabe des nichtstaatlichen Bereichs serkauftı werden.

In dem Maße, als die Grenzen nicht nur faktischer Staatsherrschaft, sondern auch des staatlichen Ordnungsmodells bewußt wurden, als somit die Begrenzung staatlicher Herrschaft zum Bestandteil der Staatsvorstellung selbst gemacht wurde, stellte sich mit Notwendigkeit die Frage, was Gegenstand staatlicher Ordnung und was im Unterschied dazu ifreic bzw. Gegenstand anderer sozialer Ordnungen sein solle. Wir wollen dies als die Frage nach den Staatsaufgaben bezeichnen. Gelegentlich ist statt dessen auch von 'Staatszwecken oder 'Staatsfunktionen, die Rede, doch haben diese Bezeichnungen einen zu hohen Bedeutungsüberschuß. So beinhaltet der Begriff der Staatszwecke eine Teleologie, deren Begründungen immer weniger zu überzeugen vermögen. Der Begriff Staatsfunktionen ist mehrdeutig und wird insbesondere auch für Teilfunktionen des Staatswesens (z.B. Legislative, Exekutive, Judikative) oder aber für die spezifische Leistung des Staates im Hinblick auf den gesamten gesellschaftlichen Zusammenhang (und unter Ausschluß der Leistungen für die Bürger oder andere gesellschaftliche Teilsysteme) verstanden. Der Terminus 'Staatsaufgaben، scheint auch deshalb geeigneter, weil er sich schon seinem alltăglichen Sinn nach auf die Frage bezieht, was der Staat soll, und zwar ohne jede essentialistische Nebenbedeutung. Staatsaufgaben können zugeschrieben, gefordert oder induktiv erschlossen werden. Diskurse über Staatsaufgaben können somit sehr unterschiedlichen Argumentationen folgen, aber es scheint nahezu unmöglich, etwas Vernünftiges über den modernen Staat zu sagen, ohne zur Frage seiner Aufgaben implizit oder explizit Stellung zu nehmen. 
Diskurse über Staatsaufgaben lassen sich nach mancherlei Gesichtspunkten ordnen. In unserem Zusammenhang sind nur staatstheoretisch bedeutsame Gesichtspunkte relevant, die also dem Staate nicht Beliebiges abfordern oder verweigern. sondern eben dies unter Berufung auf eine bestimmte Stantsvorstellung tun. Für weisen: Sie beinhalten regelmäBig zugleich Aussagen über die Aufgaben wie über die (zumindest mögliche) Leistungsfăhigkeit des Staates. Es gehört sozusagen zu den Prämissen der Vernünftigkeit derartiger Diskurse, dem Staate nichts abzufordern, was er (nach Auffassung des Fordernden) grundsåtzlich nicht kann. Wenn etwa im Anschluß an Aristoteles der Staatszweck "als Ziel der jeweiligen politischen Gemeinschaft火 verstanden wird, so ist damit vorausgesetzt, daB sich diese nicht einem unvernünftigen, also von ihr prinzipiell nicht zu realisierenden Vorhaben verschreibt. Und dort, wo aus faktischen Staatstătigkeiten der Inhalt von Staatsaufgaben abgeleitet wird, bestätigt sich die Doppelstruktur im Argument selbst: So identifiziert z.B. Heller "die Funktion des Staates * mit *der selbstandigen Organisation und Aktivierung des gebietsgesellschaftlichen Zusammenwirkens, begründet in der geschichtlichen Notwendigkeit eines gemeinsamen status vivendi für alle Interessengegensätze auf einem sie alle umgreifenden Erdgebiet * (1970: 203). Hier wird also die "immanente Sinnfunktion des Staates (ebda.) stulierten Leistung der Vermittlung zwischen demokratischen Staates und der podet.

Juristische Diskurse über Staatszwecke wollen gleichzeitig das Legitimationsproblem des Staates lösen: "Die praktische Bedeutung der Erkenntnis des Staatszweckes besteht aber darin, daß eben durch sie die psychologisch und ethisch notDeshalb werden bestimmte Staatszwecke als uird (Jellinek 1966/1900: 236). dacht, was nicht ausschließt, daß weitere, historzeitlich, begriffs notwendig gegestellt werden. Eben dies ist charakteristisch für alle rationalistiatsaufgaben festbegründungen: Die Zweckhaftigkeit des Staates begründet seinen Ordnungsspruch, wobei der Zweck eben darin besteht, eine bestimmet seinen Ordnungsanlen. Sobald politische Ordnung nicht mehr, eine bestimmte Ordnung herzustelzumindest Ermächtigung oder sonstiger als Ausdruck göttlicher Weisung oder turrechtlicher) Normen verstanden wird, mußeber (z.B. gewohnheits- oder naAnspruch der Notwendigkeit erhebt, zirkulär werden. Die Staatszwecklehren,

nem Staatsrecht und Staatswissenschaft, Grund und Angelpunkt von allgemeisind "heute zur Bedeutungslosigkeit herabgesunken" (Preu 1983.9) Stelle scheint ein auf extra-konstitutionelle Letztbegrü 1983: 9). An ihre Staatsverständnis getreten zu sein, welches die Legitimität des Stagtes aufendes che und Inhalt der Verfassung zurückführt. "Die theoretische Staates auf Tatsafall der Lehre von den Staatszwecken darf [scil. jedoch] nicht Zu und der Zerverleiten, daß im Bereich des politischen Systems die Z nicht zu der Annahme 
bracht sei oder doch ihre Bedeutung verloren habe. ... Sie besagt lediglich, daß das politische System nicht mehr durch gesellschaftlich vorgegebene, für wahr gehaltene (und damit invariante) Zwecke bestimmt werde, sondern in seiner Zwecksetzung autonom geworden ist. Nicht nur das Recht, sondern auch die Zweckaufgaben des politischen Systems sind in diesem Sinne positiviert worden: Sie werden durch programmierende Entscheidungen gesetzt, die im politischen System selbst getroffen werden müssen. (Luhmann 1968: 71).

Was als Staatsaufgabe gelten soll, läßt sich somit nicht mehr aus einem staatstheoretischen Bezugsrahmen ableiten, sondern die Entscheidung hierüber ist grundsătzlich eine Angelegenheit politischer Prozesse geworden. Zwar kamen de facto Staatsaufgaben natürlich seit jeher durch politische Entscheidungen, also durch den wie auch immer geregelten Willen der Herrschenden zustande. Neu ist jedoch, daß diesem Willen über die verfassungsmäßigen Schranken hinaus keine inhaltlichen Kriterien mehr entgegengehalten werden. Als Staatsaufgabe erscheint demzufolge, was Parlament und Regierung in einem rechtmäßigen Verfahren an Programmen auf den Weg bringen.

Vor diesem Hintergrund stellt sich die Frage, ob Inhalt und Grenzen der Staatstätigkeit eine Angelegenheit bloßer politischer Dezision geworden sind. Zwar beinhalten moderne Verfassungen nicht nur Zuständigkeits- und Verfahrensregein, sondern regelmäßig auch materiale Grenzen der Staatstätigkeit (insbes. in der Regelungen eröffnen der Politik regelmäßig einen weiten Gestaltungsspielraum. Gibt es, so ist zu fragen, Kriterien, nach denen sich Vorschläge zum Aus- oder Regelungen eröffnen der Politik regelmäßig einen weiten Gestaltungsspielraum. Gibt es, so ist zu fragen, Kriterien, nach denen sich Vorschläge zum Aus- oder Abbau der Staatstätigkeit unabhängig von partikularen Interessen oder parteipolitischen bzw. ideologischen Prăferenzen beurteilen lassen?

\section{Vier Diskurse und ihr gesellschaftstheoretischer Hintergrund}

Betrachtet man die bisherige Geschichte der Diskurse über Staatsaufgaben, also der theoretischen und politischen Erörterungen dessen, was der Staat soll oder nicht soll, so lassen sich nicht nur unterschiedliche politische Richtungen, sondern vor allem auch zeitliche Schwerpunkte bestimmter Problemstellungen identifizieren. Diese Zentrierung der Diskurse auf bestimmte Fragestellungen hängt so ist zu vermuten - mit realen Problemen der Staats- und Gesellschaftsentwicklung zusammen, so daß deren Sequenz gleichzeitig Veränderungen des Verhältnisses von sStaat und Gesellschaft anzeigt. Im folgenden sei versucht, diese Diskurse und die ihnen zugrundeliegenden Probleme aufeinander zu beziehen und im Sinne eines die kontinental-europäischen Staatsentwicklung rekonstruierenden Sequenzmodells zu interpretieren. 


\section{Polizeistaat}

Die erste Phase der europäischen Staatsentwicklung, welche etwa für die Zeit vom 16. bis zum 18. Jahrhundert anzusetzen ist, beinhaltet die Konstituierung des Staatswesens selbst als Konsolidierung der autonomen Herrschaft über ein abgegrenztes Territorium. Entscheidende Merkmale dieser Phase sind die Zentralisierung der Machtmittel und ihre Legitimation, die Trennung von Privathaushalt und Staatshaushalt der Machthaber sowie die Entstehung einer nach Aufgaben gegliederten Staatsverwaltung. Es geht hier also zuallererst um die Legitimierung, Konzentrierung und Organisierung staatsförmiger, d.h. einheitlich institutionalisierter, von der Person des Fürsten oder sonstiger Machthaber grundsätzlich zu unterscheidender, Herrschaft über ein abgegrenztes Territorium. Dementsprechend dienen Diskurse über Staatsaufgaben in dieser Epoche vor allem der Legitimation staatlicher Herrschaft. Es soll verdeutlicht werden, warum einheitliche Gebietsherrschaft notwendig ist und worin sie besteht. Preu (1983) gibt einen umfangreichen Überblick über diese Diskurse, für die die Begriffe der 'guten Polizeyı und der 'Staatszwecke` zentrale Bedeutung gewinnen.' Der Begriff als auch die Form ihrer dabei sowohl die Aufgabengebiete des Staates (policies) tion. Staatsaufgaben werden sie legitimiert. Unter den Program den Staatszwecken abgeleitet bzw. durch Sicherheit‘, 'Wohlfahrt,, ,Nützlichkeit griffe werden dabei nicht trennsichkeit` und 'Glückseligkeit‘ hervor. Diese Bezur Legitimation unterschiedlicher verwendet, sondern fungieren je nach Autor spruch einer weitgehenden, wenn nicht einer Alsen. Charakteristisch ist der Andiesen Diskursen nicht nur als Garant einer Allzuständigkeit des Staates, der in des individuellen Wohls dargestellt des kollektiven, sondern vermittelt auch Diskursen - und zwar sowohl der praxisrelevanten Literaturweise fehlt diesen tate und der Kameralisten als auch den vernunftrech Literatur der Regimentstrakdie Vorstellung eines möglichen Widerspruches lektivem Wohl und Nutzen. Der Staat wiruches zwischen individuellem und kol- als die politisch verfaßte und sich eben - der aristotelischen Tradition folgend verstanden.

Unter den erwänten Schlüsselstellung $z u$, da er sich am unen kommt demjenigen der 'Sicherheit eine stungen des entstehenden Staates bezicht. Der Begriff galt zunächst als Kon Leizu `Friede` und betraf im Spätmittelalter ver Begriff galt zunächst als Korrelat

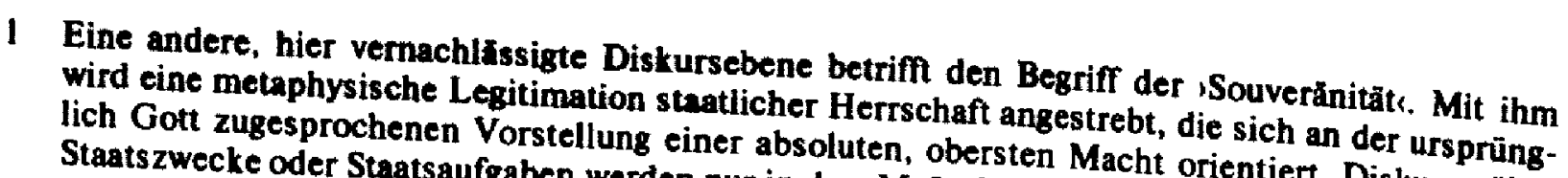
als die săkularisierte 
chen Verkehrs gegen Angriffe auf Leben und Eigentum. Mit der Konsolidierung der Territorialherrschaft entstand dann die Unterscheidung von säußerer، (Defension) und innerer Sicherheit, (Protektion). Seit Hobbes und Pufendorf ist $/ \mathrm{Si}$ cherheit ‘ zum Zentralbegriff des Staatszwecks geworden. Sie wird nicht nur als bloße Existenzsicherung, sondern als Grundlage für ein angenehmes, entlastendes, zufriedenstellendes Leben begriffen" (Conze 1984: 845). Die im 18. Jahrhundert typische Formel "gemeine Wohlfahrt und Sicherheit* läßt somit die vorherrschende Intention erkennen, "daß der Staat nicht nur als Schutzmacht, sondern auch als Wohlfahrts- und Glücksbringer durch wohlgeordnete ,Policey aufgefaßt sein sollte* (Conze 1984: 846). Die Prominenz des Sicherheitsbegriffs für die Diskurse über Staatsaufgaben bedeutet somit für die hier in Frage stehende Epoche eine retrospektive Selektion. Erst allmählich kristallisierte sich im Begriff der söffentlichen Sicherheit، die Vorstellung eines spezifischen Staatszwecks heraus, der von den Wohlfahrtszwecken analytisch getrennt und in der Folge gegen sie ausgespielt wurde. ,Öffentliche Sicherheit ‘ beinhaltet dabei sowohl die äußere und innere Sicherheit des staatlichen Schutzes von Leben und Eigentum als auch die staatliche Gewährleistung der Rechtsordnung und der daraus abzuleitenden privaten Rechte. Die Idee der offentlichen Sicherheit beinhaltet somit einen zwischen Menschen herzustellenden Zustand gegenseitiger Verläßlichkeit, in dem die mit der menschlichen 'Freiheit gegebene "double contingency of the process of interaction" (T. Parsons) aufgehoben oder zumindest auf weite Strecken berechenbar gemacht, d.h. bestimmt wird (vgl. Kaufmann 1973: 56). Die spezifische hier in Frage stehende Leistung des Staates bezieht sich somit auf die Herstellung und Gewährleistung einer Ordnung der bürgerlichen Gesellschaftr, welche ihrerseits auf den Prinzipien privater Rechte und individueller Handlungsmöglichkeiten beruht. Unter obrigkeitsstaatlichen Bedingungen galt diese Gewährleistung privater Rechte allerdings erst gegenüber anderen Privaten, nicht gegenüber dem Staate selbst. Eben dieses Problem führte zu einer grundlegenden Änderung des Diskures über Staatsaufgaben.

\section{Rechtsstaat}

In dem Maße, als sich der Obrigkeitsstaat - und hierunter sind nicht nur die Varianten des europäischen Absolutismus, sondern ebenso die republikanischen Regimente der Niederlande und der Schweiz zu verstehen - konsolidiert und die Gewährleistung der sich entfaltenden Verkehrswirtschaft übernommen hatte, wurden seine Sicherheitszwecke selbstverständlich, seine Wohlfahrtszwecke aber fraglich. Ausgelöst wurde diese Umorientierung durch den institutionellen Erfolg der konstitutionellen politischen Theorie in der amerikanischen Verfassung. Die konstitutionelle Theorie hatte ihren Ursprung in England, wo die frühzeitige Begrenzung königlicher Herrschaft die Entstehung eines absolutistischen Staatsverständnisses verhinderte und statt dessen der Vorstellung individueller Freiheitsund Widerstandsrechte zum Durchbruch verhalf. 
Die Weichen zum neuen Staatsdiskurs wurden im deutschen Sprachraum durch Kant gestellt: "Der Satz: Salus publica suprema civitatis lex est, bleibt in seinem unverminderten Wert und Ansehen; aber das offentliche Heil, welches zuerst in Betrachtung zu ziehen steht, ist gerade diejenige gesetzliche Verfassung, die jedem seine Freiheit durch Gesetze sichert; wobei es ihm unbenommen bleibt, seine Glückseligkeit auf jedem Wege, welcher ihm der beste dünkt, zu suchen, wenn er nur nicht jener allgemeinen gesetzmäßigen Freiheit, mithin dem Rechte anderer Mituntertanen Abbruch tut " (1968/1793: 154 f.). Genauer formulierte das Problem der Staatsaufgaben etwa gleichzeitig Wilhelm von Humboldt in seiner Schrift mit dem bezeichnenden Titel "Ideen zu einem Versuch, die Grenzen der Wirksamkeit des Staates zu bestimmen" (1982/1792). Er trennt nicht nur Sicherheit und Wohlfahrtszwecke (und lehnt letztere als Staatsaufgabe ab), sondern er schränkt auch Sicherheit wals der eigentliche Gegenstand der Wirksamkeit des Staats * ein auf "die Gewißheit der gesetzmäßigen Freiheit" (v. Humboldt 1982/ 1792: 115, 118) bzw. auf den Schutz der privaten Rechte.

In dieser konsequentesten liberalen Theorie der Staatsbegrenzung bleibt allerdings das Problem der strukturellen Eigenarten und Grenzen von 'Staat` und 'bür. gerlicher Gesellschaft merkwürdig unterbelichtet, obwohl sie doch der Legitimation dieser strukturellen Differenz dient. Die Staatstätigkeit wird lediglich unter dem Gesichtspunkt der Staatseinkünfte, nicht jedoch demjenigen der Staatsorganisation thematisiert. Da sich die praktisch wahrgenommenen Staatsaufgaben zumal in Deutschland entgegen den Postulaten des liberalen Staatsdenkens nur im Wirtschaftsbereich wesentlich reduzierten, im übrigen jedoch der Ausbau der Staatsverwaltung sich im 19. Jahrhundert unvermindert fortsetzte, stellte sich mit auf der Basis der konstitutionellen Iem der Kontrolle staatlicher Macht. Es wurde selseitige Kontrolle staatlicher Teilgewalten geise durch die Trennung und wechsich wechselseitig begrenzender Gewalten nur in del, doch konnte dieses System alle Staatstätigkeit an das Recht gebunden und einer richterlichen werden, als keit untergeordnet wurde. Der im Polizeistaat einer richterlichen Nachprüfbarrungssachen sind keine Justizsachen $*$ mußte beseitigt und durch die Vorstelliejustiziablen öffentlichen Rechts ersetzt werden (vgl. Grimm 1987b) Vorstellung nung von öffentlichem Recht und Privatrecht, welche für die kontinentaleuropäsche Staatstradition im Unterschied zur angelsächsischen die kontinentaleuropäiment' so charakteristisch ist (vgl. Dyson 1980), stellt die institutionelle vevern. perung der begrifflichen Differenzierung von ,Staat und institutionelle Verkörschaftı dar, welche nunmehr als Prämisse in alle weiteren Diskurgesell'Staatsaufgabenı eingeht. Die Selbstbegrenzung des Staates dient gleichzeitig seiner Verselbständigung. Sie
wird ermöglicht durch die reflexive Positivierung des Rechts, d.h. die rechtliche
Regelung seiner Änderbarkeit, sowie durch den Gedanken der Rechto keit, d.h. (neben der Gewährleistung der Privatrechtanken der Rechtsstaatlichstaatlichen Handlungen an das (öffentliche) Recht. Werdnung) die Bindung aller 
ser Staatsentwicklung hervorheben wollen, so bezieht es sich auf die Verrechtlichung aller staatlichen Vorgänge, und deren Gewährleistung. Hierauf bezieht sich die Idee der Rechtssicherheit als "das gewährleistete Vertrauen in das Bestehen des Rechts und in seine unparteiische und gerechte Handhabung" (Scholz 1955: 3).

Die Aufgabe des Staates besteht den liberalen Diskursen zufolge darin, die Freiheit und Berechenbarkeit der bürgerlichen Verkehrsbeziehungen zu gewährleisten und eben dadurch den gesellschaftlichen Fortschritt zu ermöglichen. Dagegen soll sich der Staat aller Sorge um das Glück und Wohlergehen der Bürger enthalten, da diese, sofern nur ihre Rechte gewährleistet bleiben, hierfür am besten selbst sorgen können. Dieser für das voraufklärerische Denken noch undenkbare Gedanke setzt den Glauben in die Möglichkeiten und die koordinative Leistungsfähigkeit alternativer, nicht-staatlicher Ordnungsmodelle voraus, wie er zuerst in der Denkfigur der wunsichtbaren Hand" von Adam Smith entwickelt wurde (vgl. Kittsteiner 1984). Denn daß das Zusammenwirken der Individuen ohne staatliche Vermittlung zu produktiven, für alle Beteiligten und möglicherweise sogar für unbeteiligte Dritte nützlichen Lösungen führen könne, ist für das durch Angst geprägte frühneuzeitliche Menschenbild der 'gefallenen Natur` (vgl. Delumeau 1985) unverständlich. Erst die zivilisierende Wirkung der Interessen konnte im Verbund mit der staatlichen Pazifizierung der Räume ein neues Lebensgefühl entstehen lassen (vgl. Elias 1976; Hirschmann 1980).

Die Vorstellung, daß das Geflecht der privaten Interessen - von unsichtbarer Hand gesteuert - der menschlichen Wohlfahrt dienlicher sei als staatliche Vorsorge, wird von Hegel als bürgerliche Gesellschaft im Unterschied zu Staat und Familie auf den Begriff gebracht (1968/1821: §157). Die liberale Staatstheorie nimmt nicht nur diese Unterscheidung auf, sondern postuliert eine Trennung der Sphären von Staat und Gesellschaft, die nicht nur im Postulat der Wirtschaftsfreiheit, sondern auch demjenigen der Trennung von Staat und Kirche zum Ausdruck kommt, während die von Hegel mitreflektierte Familie unberücksichtigt bleibt. Der historischen Wirkmächtigkeit dieses Gedankens hat die soziologische Gesellschaftstheorie in Theoremen der strukturellen Verselbständigung und funktionalen Spezialisierung von gesellschaftlichen Teilsystemen Rechnung getragen.

\section{Sozialstaat}

Die Eigendynamik der von staatlicher Vormundschaft mehr oder weniger befreiten bürgerlichen Gesellschaft setzte im Verein mit dem Fortschritts- und Vernunftsglauben der Epoche noch nie dagewesene Energien frei. Diese führten allerdings nicht von sich aus - wie Adam Smith angenommen hatte - zu einer gleichzeitigen Besserstellung von Reichen und Armen, sondern zu neuen Strukturen sozialer Ungleichheit, die sich im wesentlichen am Besitz oder Nicht-Besitz von Produktionsmitteln festmachten. Deutsche Beobachter der vorauseilenden englischen und französischen Entwicklung diagnostizierten schon vor der Mitte des 
19. Jahrhunderts den nicht nur vorübergehenden, sondern strukturell angelegten Charakter der bestehenden Formen von Ausbeutung und Armut und entlarvten damit die liberale Vision einer heraufkommenden Gesellschaft bürgerlicher Eigentümer als Illusion. Während Engels und Marx davon ausgingen, daß der Staat als Instrument der herrschenden Klassen durch Unterdrückung der aufbegehrenden Massen die Ausbeutung auf die Spitze treiben und eben dadurch der Revolution den Weg bereiten müsse, sah Lorenz von Stein trotz ähnlicher Diagnose des Klassenantagonismus den Staat als eben jene Macht, die in der Lage sei, als ssozialer Staatı durch Einführung des allgemeinen Wahlrechts und die Schaffung einer ssozialen Verwaltung die Bedingung eines antagonistischen Klasseninteresses zu verändern und dadurch sowohl bei der besitzenden wie bei der nicht-besitzenden Klasse die Einsicht in den teilweise komplementären Charakter ihrer Interessen zu fördern (vgl. Böckenförde 1976c). Damit wurde bereits früh ein Grundgedanke formuliert, der erst im Laufe des 20. Jahrhunderts zu einer Reformulierung des Staatsverständnisses führen sollte. Es ist dies der Gedanke einer staatlich induzierten Sozialreform, der - wenngleich mit anderen Akzentsetzungen - auch in Frankreich (durch Sismondi) und in England (durch Bentham und J.S. Mill) zuerst Wie bereits erwähnt, hat sich das praktische Politik-bzw. Polizey-Verständnis nie an die engen Grenzen gehalten, die ihm von der liberalen Staatstheorie gezogen wurden. So entstanden in den meisten europäischen Staaten Gesetze und MaBnahmen, um die Folgeprobleme der Industrialisierung und Verstädterung unter Kontrolle zu halten. Diese Folgeprobleme wurden jedoch zunächst recht unterschiedlich formuliert, und dementsprechend unterschieden sich auch die zeitlichen Prioritäten der sozialpolitischen Maßnahmen sowie deren Implementation erheblich in den verschiedenen Staaten Europas (vgl. Alber 1982; Ashford 1986; Kaufmann 1986: $71 \mathrm{ff}$.). Zumeist handelte es sich zunächst um recht punktuelle Interventionen, die jedoch im Laufe der Zeit an Intensität und Reichweite zunahmen und zur Entstehung spezialisierter Fachverwaltungen und vielfach auch zu schaft führten. Charakteristische Organisationsformen dieser Art finden Gesellbesondere im Bereich der Maßnahmen der Einkommenssicherung finden sich insArbeitsfahigkeit oder Arbeitsmöglichkeit (soziale Sicherung) Entwicklung der Dienstleistungen des Bildungs-, Gesundheits- und Sozialwesens Nachdem die Weltwirtschaftskrise die Fragilität ausschließlich marktgesteuerter Versorgungsmodi offenkundig gemacht und die Alliierten in der Atlantikcharta von 1941 sich auf eine wohlfahrtsstaatliche Verantwortung festgelegt hatten, brachte die lange Periode wirtschaftlicher Prosperităt nach dem Zweiten WeltEuropas (vgl. Flora 1986 ff.).

Diese De-facto-Entwicklung wurde erst sehr allmählich dem staatstheoretischen Verstandnis erschlossen. Zwar bürgerte sich neben dem Begriff der Sozialpolitik
der schon 1876 von Adolph Wagner benutzte Begriff des, Wohlfahrtsstaats in der 
politischen Sprache der Weimarer Republik ein, doch fehlte es trotz der im wesentlichen auf den 'KlassenkompromiB, des IStinnes-Legien-Abkommenss vom 15.11.1918 zurūckgehenden sozialstaatlichen Programmatik der Weimarer Reichsverfassung an einer entsprechenden staatsrechtlichen Durchdringung. Da die sozialen Grundrechte „dem Staat nicht wie die klassischen Grundrechte Schranken zogen, sondern ihn zu sozialem Handeln anhielten, erschienen sie nicht direkt anwendbar, sondern bedurften gesetzgeberischer Vermittlung. Die Staatsrechtslehre war aber nicht bereit, sie dann wenigstens als verfassungsrechtliche Zielvorgaben für die Gesetzgebung und Auslegungshilfen fur die Gesetzesanwendung zu betrachten, sondern erklärte sie kurzerhand zu Nicht-Recht. Sie erschienen auf diese Weise als bloße Absichtserklărungen, die in der Verfassung standen, ohne an deren normativer Geltung zu partizipieren* (Grimm 1987a: 155). Auch nach dem Zweiten Weltkrieg gewann die Sozialstaatsklausel des Grundgesetzes erst allmăhlich verfassungsrechtliche Konturen (vgl. Forsthoff 1968; Häberle 1972), während nunmehr der Begriff des ,Wohlfahrtsstaats، polemisch zur Abgrenzung von den am Prinzip der Staatsbürgerversorgung orientierten englischen und skandinavischen 'welfare states, verwendet wurde. Erst seit etwa einem Jahrzehnt werden - nicht zuletzt unter dem Gesichtspunkt des im Zuge der europäischen Einigung immer wichtiger werdenden intemationalen Vergleichs - die Begriffe Sozialstaat und Wohlfahrtsstaat weitgehend synonym verwendet.

Die neuere verfassungsrechtliche Interpretation in der Bundesrepublik bezeichnet die Aufgabe der Sozialstaatlichkeit als Gewahrleistung der wsozialen Voraussetzungen der Realisierung grundrechtlicher Freiheit* (Bockenforde 1976b: 238) und sucht, durch Bezugnahme auf die Grundintention des liberalen Staatsverstăndnisses, die zunächst in den Vordergrund gestellte Spannung zwischen Sozial- und Rechtsstaatlichkeit zu úberwinden. In Verbindung mit weiteren Staatszielbestimmungen werden so die verfassungsmäßigen Konturen einer Gesellschaftsordnung konstruiert, woraus sich zwar keine konkreten Rechtsanspriche und auch keine verbindlichen Auftrige an den Gesetzgeber, aber doch Beurteilungsgesichtspunkte und Auslegungskriterien hinsichtlich der Gesetzgebung ableiten lassen.

Die Ausformulierung der sozialstaatlichen Aufgaben erfolgte somit nicht auf der Verfassungs-, sondern auf der Gesetzgebungsebene (vgl. Zacher 1980). Und hier zeigen sich weiterhin charakteristische Unterschiede hinsichtlich des Inhaltes und der Implementationsbedingungen der neuen Rechtsmaterie. Zum einen fuhren sie zu einer Aufblahung und fortschreitenden Differenzierung des Verwaltungsrechts, wobei die Unterscheidung von Eingriffs- und Leistungsverwaltung Bedeutung gewinnt. Diese orientiert sich an der idealtypischen Gegenüberstellung von Rechtsund Sozialstantlichkeit und reproduziert sie auf verwaltungsrechtlicher Ebene. Idealtypisch lassen sich Eingriffe, die ihre Grenzen an privaten Rechten finden, unterscheiden von gewăhrenden Formen staatlichen Handelns. Aber diese Unterscheidung deckt sich nicht mit derjenigen von Ordnungsgewhilleistung und So- 
zialgestaltung, da beispielsweise die Erhebung von Sozialversicherungsbeiträgen oder die Gewerbeaufsicht durchaus der Eingriffsverwaltung zuzurechnen sind. Zudem stellen natürlich auch Leistungsgewährungen - beispielsweise Subventionen oder das Angebot von Kindergärten - Interventionen in gesellschaftiche Zusammenhänge (hier der Wirtschaft bzw. der Familie) dar, wie zuerst die natio. nalökonomische Analyse des Interventionismus (vgl. Küng 1956) verdeutlicht
hat.

Die entscheidende Differenz zwischen der älteren Auffassung von Rechtsstaatlichkeit und der neueren einer sozialen Rechtsstaatlichkeit bezieht sich auf das Verhältnis von Staat und den übrigen Lebensbereichen, der sogenannten ,Gesellschaft 1 . Der Staat kann nun nicht mehr als bloßer Garant einer grundsãtzlich privatrechtlich geordneten und insoweit von ihm getrennten, Gesellschaft fungieren, sondern seine Maßnahmen sind ihrem Sinn nach auf die Beeinflussung der gesellschaftlichen Verhältnisse gerichtet, allerdings nicht mehr in dem umfassenden Sinne, den der alte Obrigkeitsstaat beanspruchte. Die strukturelle Verselbstăndigung der gesellschaftlichen Teilbereiche und die grundsātzlich unabhăngige Rechtstellung der in ihrem Kontext handelnden individuellen und kollektiven Akteure wird anerkannt, doch wird versucht, unerwinschte Folgen der daraus resultierenden Eigendynamik zu korrigieren oder zu kompensieren. Derartige unerwünschte Folgen werden als soziale und/oder politische Probleme thematisiert, also in einer bestimmten Weise gedeutet, um die Staatsmacht zu ihrer Lösung in Bewegung zu setzen. Dabei werden die Folgen typischerweise an bestimmten, als benachteiligt oder sozial schwach definierten Bevölkerungsgruppen festgemacht, zu deren Gunsten der Staat im Namen der von ihm repräsentierten Gerechtigkeit tâtig werden soll. Allerdings findet solche Staatstătigkeit - dem wirtschaftspolitifunktionsfähigen Wettbewerbs ihre Grenzen (vgl. Blum 1980). Aus gesellschaftstheoretischer Sicht läßt sich ein zentrales Folge.

nåchst mit dem Begriff der Inducht läßt sich ein zentrales Folgeproblem der zuModernisierung angesprochenen gesellsung, heute zumeist mit dem Begriff der bensbedingungen der Individuen gesellschaftlichen Verānderungen für die LeParsons 1972: $32 \mathrm{ff}$. Lividuen mit dem Begriff der Inklusion ansprechen (vgl. Zuge der strukturellen systeme die alten Sorgeverbänderenzierung funktional spezialisierter Handlungswenngleich bescheidenem und je sich aufgelöst haben, die den Individuen auf dennoch die Befriedigung der wesentlozialem Stand unterschiedlichem Niveau dere die ganze Lebenssituation des Indichen Bedürfnisse sicherten und insbesonder Gesellschaft auf funktional des Individuums im Blick hatten. Mit dem Umbau sammenhängen eben diese Ganzezialisierte Strukturen gerät in den sozialen $\mathrm{Zu}$ Blick, so daß es nunmehr dem Individuum Lebenssituation der Individuen aus dem sehr unterschiedlichen Systemzudividuum selbst obliegt, durch Beteiligung an dige und Lebensdienliche $z u$ besammenhängen sich das für es Lebensnotwenjedermann sicherzustellen wird beschaffen. Solche soziale Teilhabemöglichkeit für 
Politik, die sich dazu insbesondere des Instruments der Definition sozialer Rechte bedient. Dieser Gedanke lag ursprünglich auch der Forderung nach sozialer Sicherheit zugrunde, bis dieser Begriff im Fortgang der Sozialpolitik auf die Teildimension der Einkommenssicherung reduziert wurde (vgl. Kaufmann 1973, bes. 92 ff., 169 ff., 208 ff.).

Die Legitimation für sozialgestaltende Eingriffe liegt somit nicht in einem allgemeinen Ordnungsinteresse, sondern in den spezifischen Wirkungen, die von ihnen erwartet werden. Zwar kann im politischen Prozeß über diese Wirkung selbst nicht entschieden werden, die Wirksamkeit oder Unwirksamkeit von Maßnahmen sowie deren oft unvorhergesehene Nebenwirkungen erweisen sich erst später im Prozeß der Implementation und deren Folgen. Und nicht selten scheinen politische Maßnahmen sogar mit der Vermutung (oder von interessierter Seite sogar in der Hoffnung) auf ihre Unwirksamkeit beschlossen zu werden, um zumindest den Anschein politischer Problembekämpfung zu erwecken (vgl. Edelman 1976). Dennoch kommen politische Diskurse, welche sozialgestaltende Maßnahmen des Staates fordern, nicht umhin, die Probleme zu benennen, für die Abhilfe gefordert wird, um die Wirksamkeit der vorgeschlagenen Maßnahmen zu behaupten. Die Forderung an den Staat zum Eingreifen kann hier im Regelfall nicht mit Rechtsverletzungen begründet werden, sondern sie bedarf der Behauptung einer spezifischen Leistungsfahigkeit des Staates zur Änderung bestimmter Zustände oder zur Lösung bestimmter sozialer Probleme. Häufig bleibt diese Behauptung allerdings dem Diskurs implizit: Das politische Argument beschränkt sich auf die Dramatisierung des Problems und den Appell an die Verantwortlichkeit des Staates, der um so plausibler erscheint, je stärker Staatsorgane mit Bezug auf bestimmte Probleme bereits tătig geworden sind. Dennoch bleibt auch hier die erstrebenswerte Wirkung - häufig als ,Ziel einer Maßnahme، bezeichnet - der rationale Kern des Arguments.

Es bedarf allerdings in der Regel eines erheblichen Problemdrucks, um staatliche Instanzen zu neuen Eingriffen zu veranlassen, die ja in allen gravierenden Fällen umstāndliche Verfahren der Rechtssetzung und - soweit die Eingriffe oder Leistungen umstritten sind - zusätzliche justizielle Kontrollen auslösen. Nicht nur die Knappheit staatlicher Mittel, sondern auch die Knappheit der für politische Prozesse benötigten Zeit und Aufmerksamkeit schränken das Interventionspotential des Staates ein und üben einen $\mathbf{Z}$ wang zur politischen Prioritätenbildung aus, die sich weniger nach Sachgesichtspunkten denn nach Interessenlagen und politischem Einfluß vollzieht. Nur unter dem Druck der anwachsenden Arbeiterbewegung hat sich in Europa die Sozialpolitik entwickelt und dadurch den Kapitalismus transformiert. Dies geschah allerdings nicht allein durch diesen Druck und die damit verbundene Änderung der Interessen der Herrschenden, sondern gleichzeitig im Kontext der Ideen von Freiheit und sozialer Gerechtigkeit, deren geistiger Hintergrund - Christentum und Aufklärung - herrschende Klassen und soziale Bewegungen verband (Heimann 1980/1929).

Die nunmehr als grundsätzlich legitim geltende 'Einmischung des Staates in die 
gesellschaftlichen Verhältnisse verändert das Verhältnis von Staat und Gesellschaft sowohl auf der Seite des Policy-Input wie des Policy-Output. Da sozialgestaltende Staatseingriffe notwendigerweise auf konstituierte Interessenlagen treffen, entsteht bei den davon virtuell Betroffenen ein politisches Interesse, auf die staatlichen Willensbildungsprozesse und Entscheidungen EinfluB zu nehmen. Deshalb formieren sich Akteure mit ähnlichen Interessen zu Verbānden, welche auf verschiedenen Wegen um politischen Einfluß ringen (vgl. Ritter 1988). Dadurch wird die idealtypische Trennung von Staat und Gesellschaft durchbrochen; gesellschaftliche Kräfte suchen unmittelbar Einfluß nicht nur auf das Parlament, sondern auch auf die Staatsadministration zu gewinnen.

Aber auch auf der Seite staatlichen Handelns wird die Grenze zwischen 'Staat، und 'Gesellschaft verwischt: Bei der Beeinflussung der gesellschaftlichen Verhältnisse bedient sich der Staat in zunehmendem Maße nichtstaatlicher Akteure, sei es durch die Schaffung öffentlich-rechtlicher oder selbst privatrechtlicher Einrichtungen (Schuppert 1981; Hood 1986), sei es durch Ermächtigung und Verpflichtung sowie Subventionierung genuin 'gesellschaftlicher (Akteure wie Unternehmungen oder Verbände. Schließlich ergibt sich auch auf der Ebene der LeiGeldpolitik und Zuteilungspolitik. Sie treffen der Steuerpolitik, Haushaltspolitik, bar mit Gebot oder Verbot, sondern indirekt, durch Anreize, Erleichterungen vermehrte oder geminderte Zuteilung; sie entziehen sich daher nahezu ganz der

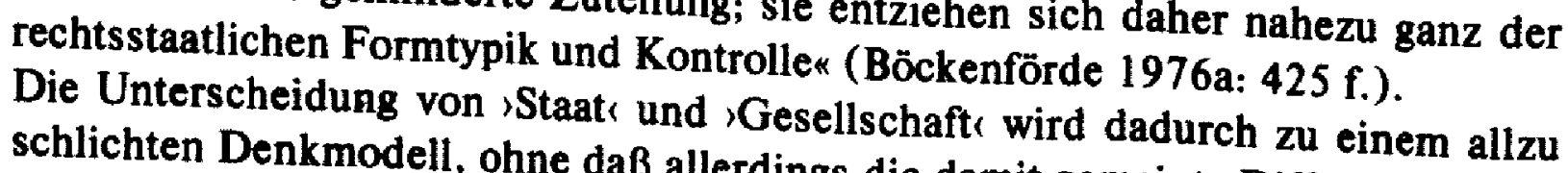
sätzlich aufgehoben würde (vgl. III.).

\section{Steuerungsstaat}

Obwohl die staatstheoretische Verarbeitung der wohlfahrtsstaatlichen Entwicklung noch keineswegs an ihr Ende gelangt ist, scheint sich in jüngster Zeit der Diskurs über Staatsaufgaben auf neue Fragen und Problemstellungen zu verschieben, wie nicht zuletzt zahlreiche Beiträge in diesem Band belegen. Es ist immer bezeichnen, als das Werdende. Ausgeformte zu beschreiben und eindeutig zu vierte Phase der Diskurse über Staatsaufgaben zu postulieren fruchtbar, eine stika zu beschreiben zu versuchen. Dadurch zann postulieren und ihre Charakterijüngsten Diskussion, sondern auch das davon abzuhebende Charakteristische der vorangehenden Phase noch verdeutlicht werden. Die neuen Trends politischer Anforderungen an folgt skizzieren. 
1. Offenkundig werden neue Staatsaufgaben im Bereich des Schutzes natürlicher Ressourcen formuliert. Nicht der ,Umweltschutz ist neu, die Stadtsanierungspolitik der frühen Neuzeit lag durchaus auf dieser Linie. Aber das Konzept der Umwelt hat sich gewandelt, es ist international, ja tendenziell global geworden, insbesondere im Hinblick auf mögliche Klimakatastrophen. Nicht mehr die durch Umweltverschmutzung gefăhrdeten Individuen oder sozialen Gruppen stehen im Vordergrund des Interesses, sondern sdie gefährdete Natur,, welche in der Form von durch menschliche Handlungen gefährdeten ökologischen Systemen vorgestellt wird. Dem Staat wird in diesem Zusam. menhang zugemutet, das Verhalten von Individuen und Wirtschaftsorganisationen so zu beeinflussen, daß der notwendige Schutz der Umwelt und die Exitenzgrundlagen zukünftiger Generationen gewährleistet Jwerden (vgl. hierzu die Beiträge von Kirchgässner und Windhoff-Héritier in diesem Band).

2. Zunehmend verschieben sich sozialpolitische, aber auch kriminalpolitische und wirtschaftspolitische Ansprüche aus dem Bereich des kontrollierenden, korrigierenden und kompensierenden Eingreifens in denjenigenn des präventiven Eingreifens. Dem Staat wird die Fähigkeit zugesprochen - oder abgefordert - durch seine Maßnahmen nicht nur Schaden abzuwehren oder seine Folgen zu beseitigen, sondern der Entstehung von Schäden durch die Intervention im Bereich der Gefahrenquellen oder -ursachen vorzubeugen (vgl. Grimm 1986; Preuß in diesem Band). Dies äußert sich im Bereich der Sozialpolitik z.B. durch die wachsende Bedeutung qualifizierender, beratender oder präventiver sozialer Dienstleistungen sowie überhaupt durch eine Gewichtsverlagerung von den Geldtransfers zu den als gestaltungswirksamer,angesehenen Realtransfers. Im Bereich der Verbrechensbekämpfung wird präventives Eingreifen zur Verbrechensverhinderung zunehmend als Königsweg gepriesen. Und im Bereich der Wirtschaftspolitik tritt an die Stelle der Forderung nach einer die Eigendynamik der Konjukturen korregierenden Globalsteuerung die Forderung nach vorausschauender Wirtschaftsförderungs- und Technologiepolitik (vgl. den Beitrag von Kitschelt in diesem Band).

3. Weniger in der politischen Praxis als in den rechts- und staatstheoretischen Diskursen werden neue Formen staatlichen Handelns erörtert und zum Teil gefordert. Den Ausgangspunkt bilden dabei Diagnosen über unerwünschte Nebenfolgen oder auch mangelnde Effektivität herkömmlicher, insbesondere hoheitlicher Formen staatlichen Handelns für die Lösung aktueller politischer Probleme. Diagnostiziert wird ein 'Steuerungsdefizit/ des Staates, das allerdings unterschiedlich eingeschätzt wird. Während Staatsrechtler überwiegend die Erosion der hoheitlichen Stellung des Staates und seine wachsende Abhängigkeit von der Kooperationswilligkeit geselischaftlicher Akteure beklagen und das zunehmende Leerlaufen rechtsstaatlicher Kontrollformen vor den neuen Staatsaufgaben befürchten (vgl. Böckenförde 1976d; Grimm 1986), neigen Sozialwissenschaftler dazu, problematische Nebenwirkungen hoheitlicher Formen staatlicher Steuerung und neue, nicht-hoheitliche Steuerungsmöglichkeiten 
des Staates aufzuzeigen (vgl. Kaufmann 1982; Mayntz 1983; Dahme/Grunow 1983; Glagow 1984; Kaufmann/Majone/Ostrom 1986; Teubner 1989; Schuppert und Willke in diesem Band).

4. Souveränitätsverluste des Staates werden nicht nur mit Bezug auf seine innere, gesellschaftliche Umwelt diagnostiziert, sondern auch im Hinblick auf den Charakter der internationalen Beziehungen. Zwar bleiben Staaten nach wie vor die entscheidenden völkerrechtlichen Subjekte, aber sie sind dies nicht mehr unter der Prämisse von Autarkie, sondern mehr und mehr unter der Prämisse des Eingebundenseins in internationale und zum Teil supranationale Zusammenhänge. Die gesellschaftlichen Zusammenhänge überschreiten in wachsender Intensität die nationalstaatlichen Grenzen, die Interdependenz zwischen den Staaten wächst und tendiert zur Weltvergesellschaftung (vgl. Luhmann 1975; Bornschier 1980; Heintz 1982; Bornschier/Lengyel 1990). Es sind also nicht bloß die mehr oder weniger freiwillig eingegangenen völkerrechtlichen Verpflichtungen, sondern durchaus unfreiwillige Abhängigkeiten von weltweiten Entwicklungen wie z.B. der Weltkonjunktur, dem Ölpreis oder der Technologiepolitik bestimmter Drittländer, welche sich - vermittelt über die Entwicklung von Wirtschaftswachstum und Vollbeschäftigung - auch direkt auf die Staatsaufgaben und die Staatshaushalte auswirken. Diese Zusammenhänge sind zwar nicht grundsätzlich neu, aber sie scheinen sich fortschreitend zu intensivieren, und sie sind erst in jüngster Zeit bewußtseinsbestimmend geworden. Dies veranlaßt manche Staaten oder auch supranationale Zusammenschlüsse wie die EG zu einem ineuen Merkantilismusı, indem durch gezielte staatliche Vorgaben der Wirtschafts- und Technologieförderung die internationale Wettbewerbsstellung zu verbessern gesucht wird (vgl. Willke 1988; Willke
1991).

Zwischen diesen Entwicklungstendenzen sind Zusammenhănge zu vermuten. Die neuen Staatsaufgaben lassen sich nämlich gerade dadurch charakterisieren, daß sie besonders deutlich die Inadäquanz oder zumindest Ergänzungsbedürftigkeit des herkömmlichen Verständnisses vom staatlichen Handeln aufweisen. Symptomatisch scheint hierbei die Begriffsverschiebung von Intervention zu iSteuerung: Nicht mehr bloB mehr oder weniger punktuelle oder lokale Interventionen des Staates zur Korrektur von Rechtsverletzungen, zur Gefahrenabwehr oder zur Besserstellung bestimmter Bevölkerungsgruppen werden gefordert, sondern ein sysiembeeinflussendes Handeln, welches somit nicht mehr unmittelbar bei der Sanktionierung des Handelns einzelner Akteure, sondern bei ihren Handlungsprä-
missen ansetzt.

Zum Verstăndnis dieser Problemverschiebung muB weniger auf staatstheoretische denn auf gesellschaftstheoretische Einsichten rekurriert werden. Was die älteren Staatswissenschaften schlicht als ,Gesellschaft, (nämlich: nicht-Staat!) bezeichneten, stellt sich heute zunehmend nicht mehr nur der Soziologie, sondern 
auch dem öffentlichen Bewußtsein als ein institutionell vielfältig gegliederter, überwiegend hoch organisierter und aus systemtheoretischer Perspektive besser verständlicher Raum sozialer Beziehungen oder Kommunikationen dar. Die in den älteren Theorien (mit Ausnahme der Markttheorie!) lediglich implizite Annahme emergenter Ordnungen auch im nicht-staatlichen Bereich (Smiths Innsichtbare Hand,) wird nunmehr reflexiv verarbeitet. Eben deshalb wird nun dem Staat zugemutet, seine Einflußnahmen auf die durch seine Rechtsordnung mitkonstituierten sozialen Verhältnisse als Systemsteuerung und nicht als bloße Intervention zu konzipieren. Denn da alle Akteure in sozialen Systemen unter den Prämissen dieser Systeme handeln, können punktuelle Interventionen von außen im Regelfalle nur durch derartige Systemeigenschaften igebrochen (wirksam werden. Das gilt insbesondere angesichts des Umstandes, daß die Adressaten staatlicher Steuerungsleistungen zunehmend kollektive, also organisierte Akteure sind, die ihre Existenzmöglichkeit der staatlichen Rechtsordnung verdanken. Sie stehen unter spezifischen organisationsinternen und institutionellen Restriktionen, welche ihre Leistungen grundsätzlich als berechenbarer erscheinen lassen als diejenigen von Individuen (vgl. Mayntz u.a. 1988).

Das Neuartige der Zumutung an die Art der staatlichen Aufgabenfüllung besteht also darin, daß diese nicht einfach und allein von der ihr durch das traditionelle Staatsverständnis zugesprochenen hoheitlichen Position in die 'gesellschaftlichen Verhältnisse interveniert, sondern die spezifischen Eigenarten des jeweiligen Interventionsfeldes und die Interessen der betroffenen Akteure als notwendige Wirkungsbedingungen der eigenen Intervention reflektiert. Da hoheitliches Handeln auf der Basis von Geboten und Verboten notwendigerweise durch eine hohe Inflexibilităt charakterisiert ist und daher zu Abwehr - und Vermeidungsstrategien unliebsam betroffener Akteure besonders einlädt, da also regulative Politik in bestimmten Gegenstandsbereichen wie den sozialen Diensten, dem Umweltschutz oder der Wirtschaftsförderung häufig kontraproduktive Wirkungen zeitigt, werden dem Staat ,weicheres Strategien, wie Anreizprogramme, Überzeugungsprogramme (vgl. Mayntz 1983), die Konstituierung von Verhandlungssystemen, 'prozeduralesı bzw. rreflexives Recht، oder ,Kontextsteuerung، (vgl. Teubner/Willke 1984), empfohlen.

Diese Beschreibung der neuen Diskurslage macht allerdings noch nicht recht deutlich, wodurch der Themenwandel bedingt ist. Man kann ihn historisch um die Mitte der 70er Jahre datieren, als durch den ersten Bericht des Club of Rome zur Lage der Menschheit* (Meadows u.a. 1972) sowie die anschließende Ö̈lkrise، erstmals die globale Verflechtung moderner Staaten und ihrer Bewohner auf breiter Ebene bewußtseinsfahig wurde. Die anschließende wirtschaftliche Rezession brachte zudem die fiskalischen Grenzen wohlfahrtsstaatlicher Politik ins politische Bewußtsein, und etwa gleichzeitiz wurden auch andere Grenzen staatlicher Problemlōsungsfahigkeit als, Krise thematisiert. Nachdem jedoch die meisten westlichen Staaten die offenkundigen Defizite ihre Leistungsfahigkeit mit bemerkenswerter Robustheit überstanden haben, ist sozialwissenschaftliche Krisenrhe- 
torik bald durch komplexere Theorien der sozialen Zusammenhănge obsolet geworden.

Dennoch hat sich das offentliche Unbehagen nicht beruhigt. Im Begriff des, Risikos، oder gar der ,Risikogesellschaft، (Beck 1986) werden Eigenschaften moderner Sozialzusammenhänge thematisiert, welche deren Eigendynamik als Selbstund Fremdgefährdung deuten, und eben hiergegen wird, Risikovorsorge als Staatsaufgabe" (so Preuß in diesem Band) gefordert.

Vorsorgende Gefahrenabwehr - also Prävention - ist, wie PreuB zeigt, ebenso wenig eine prinzipiell neue Zumutung an die Staatstătigkeit wie der erwăhnte Umweltschutz. Insofern würde die Bezeichnung der geforderten neuen Staatsqualităt als $>$ Präventionsstaatlichkeit ${ }^{2}$ am Problem vorbeigehen. Der Kern des Problems liegt nämlich darin, daß Prävention mit Bezug auf bestimmte Risiken unmóglich ist, weil wir für bestimmte, als durchaus real anzusehende Gefahren über keine ausreichenden Erklärungsmodelle verfügen. "Globaleffekte und Überraschungseffekte lassen sich, wenn man realistisch bleiben will, schwer auf Einzelentscheidungen zurechnen ... mag der Entscheider so gut kalkulieren wie er will: man kann wissen (und er kann wissen), daß sich beim Zusammenwirken vieler Entscheidungen deren Gesamteffekte und deren überraschende Koinzidenzen jeder Prognose entziehen (Luhmann 1990: 41). Diese beunruhigende Perspektive, daß die nErzeugung unvorhersehbarer [scil. möglicherweise weitreichende Schäden verursachender] Ereignisse als eine systematische Eigenschaft der gesellschaftlichen Entwicklung* (Preuß in diesem Band: 534) anzusehen ist, führt insofern mit guten Gründen zu Anforderungen an den modernen Staat, als ein kollektiver Verzicht auf das Eingehen bestimmter Risiken mit unabsehbaren Folgen nur durch kollektiv bindende Entscheidungen erfolgen kann.

Die neuartige Zumutung, welche aus dieser Problemlage an den Staat erwăchst, bezieht sich somit nicht mehr nur auf die Verantwortung für die Einhaltung des Rechts (wie im Diskurs der Rechtsstaatlichkeit) oder für die Gewährleistung individualisierbarer Wohlfahrt der Bürger (wie im Diskurs der Sozialstaatlichkeit), sondern auf die Verantwortung für die Gewährleistung des unschädlichen Zusammenspiels der Eigendynamik verschiedener sozialer Systeme (vgl. Willke in diesem Band). Dem Staat wird also eine Steuerungsleistung für diese Systeme in der Weise zugemutet, daß zwar nicht deren als notwendig erachtete Selbststeuerungsfähigkeit in Frage gestellt wird, aber die externen Folgen ihrer Eigendynamik in Grenzen gehalten werden. Ich schlage daher zur Kennzeichnung dieses Diskurses
über Staatsaufgaben den Begriff des 'Steuerungsstaates` vor. Als Steuerungsaufgaben lassen sich solche Erwartungen an die Staatstätigkeit bezeichnen, welche das Verhältnis von Staat und den sonstigen gesellschaftlichen Funktionsbereichen nicht mehr als ein Gegebenes, sondern als ein reflexiv stets erneut durch politische Entscheidung zu Gestaltendes thematisieren. Praktischer

2 Diesen Vorschlag machte ich anläßlich meiner Prásentation im Rahmen der ZiF-Forschungs-
gruppe. 
Ausdruck dieser staatlichen Gestaltungsmacht ist der $\delta$ ffentliche Sektor als strukturell differenziertes Ergebnis staatlicher Einzelpolitiken wie z.B. der Gesundheits-, Bildungs-, Wissenschafts- oder Technologiepolitik. Eine Vielzahl nichtstaatlicher Akteure wirken hier unter der Prämisse staatlich gesetzten Organisations- und Verfahrensrechts sowie z.T. staatlich geregelter Finanzierung zusammen, um bestimmte $Z$ wecke zu verfolgen. Inhalt und Legitimation dieser sektoralen Politiken gehen nicht ausschlieBlich vom Staat aus, sondern entstehen in fortgesetzten Aushandlungsprozessen zwischen den beteiligten Akteuren. ${ }^{3}$ Die entsprechenden Willensbildungsprozesse vollziehen sich zumeist im vorparlamentarischen Raum, bleiben aber - um verbindlich zu werden - auf demokratische Legitimation angewiesen. Insofern ist der Staat nicht bloB sprimus inter pares* (Willke) oder gar bloß Notar der von den gesellschaftlichen Akteuren gefundenen Vereinbarungen. Zwar sind die Machtverhältnissen zwischen den staatlichen und den nichtstaatlichen Arteuren von Land zu Land und von Politikbereich zu Politikbereich verschieden, aber im Regelfall würden die gesellschaftlichen Akteure ohne die staatliche Verfahrenssteuerung - und nicht selten ohne staatlichen Druck - überhaupt nicht zu langfristig wirksamen Vereinbarungen kommen. Andererseits ist es dem Staat aus vielfältigen Gründen (vgl. hierzu zahlreiche Beiträge in diesem Band, systematisch insbesondere Schuppert) nicht möglich, mit Bezug auf die anstehenden komplexeren Steuerungsprobleme aus eigener Machtvollkom. menheit zu entscheiden. Die Erhöhung staatlicher Steuerungskapazität setzt hier neue, lernfähigere Formen politischer Willensbildung voraus (vgl. Preuß in diesem Band).

\section{Folgerungen}

Gegenstand dieser Überlegungen ist nicht die reale Entwicklung des Staates, sondern die Sequenz typischer Diskurse über die Aufgaben des Staates. Zwar gibt es gute Gründe für die Annahme, daß diese veränderten Diskurse auch Veränderungen der faktischen Staatstätigkeit reflektieren, aber diese Veränderungen sind bei weitem nicht so grundsătzlich wie die Differenzen der staatstheoretischen Diskurse. Das Auftreten eines neuen Diskurses über die Aufgaben des Staates bezieht sich stets auf den bereits erreichten Stand staatlicher Aufgabenerfüllung, der dadurch nicht grundsătzlich in Frage gestellt, aber relativiert wird. Neue Diskurse über Staatsaufgaben reflektieren Veränderungen der Auffassung über das Verhältnis zwischen dem Staat und dem Wirkungsfeld staatlicher Tătigkeit.

3 Die Struktur der diesen Aushandlungsprozeß tragenden Beziehung variiert je nach politischem System. In zahlreichen Staaten Kontinentaleuropas scheint ein korporatistisches Arrangement vorzuherrschen, d.h. ein relativ enges Netzwerk zwischen den für bestimmte Teilpolitiken zuständigen staatlichen Stellen und gewissen privilegierten Akteuren; dagegen erscheinen die diesbezüglichen Netzwerke in den Vereinigten Staaten locker und für Dritte zugánglicher (vgl. Windhoff-Héritier in diesem Band). 
Wăhrend der polizeistaalliche Diskurs die Gesamtheit der Lebensverhaltnisse im Herrschaftsgebiet zum Gegenstand staatlicher Vorkehrungen machte, reduzierte der rechtsstaatliche Diskurs die Stantstătigkeit auf die Gewihrleistung des außeren Schutzes und der Rechtsordnung als Grundlage der grundaltzlich freien Entfaltung aller Būrger. Das Wirkungsfeld des Staates beschrlnkt sich hier auf die Setzung. Handhabung und Durchsetzung des Rechtes, whrend die ubrigen Sozialverhältnisse - in der Restkategorie ,Geselischaft، zusammengefaBt - grundsảtzlich nicht als Gegenstand staatlicher Einwirkungen gelten. Dieses reduktionistische Verständnis der Staatsaufgaben ist als Reaktion auf die undifferenzierten Allzustāndigkeitsprärogativen des polizeistaatlichen Diskurses verstindich. Der sozialstaatliche Diskurs entfaltete sich sodann in kritischer Auseinandersetzung mit den liberalen Prämissen des rechtsstaatlichen Diskurses. Er konzipiert als Wirkungsfeld staatlicher Tătigkeit die sozialen Verhältnisse und die Lebenslage der Bürger, wobei es primär um die Minderung von Differenzen sozialer Macht und um den Ausgleich sozialer Benachteiligungen geht, welche aus dem ifreien Spiel der gesellschaftlichen Kräfter resultieren. Diese Ziele sollen durch die Einräumung von Schutzrechten sowie durch staatliche oder staatlich vermittelte Leistungen an Individuen abgebaut werden. Der steuerungsstaatliche Diskurs schließlich resultiert aus der Einsicht, daß Rechtssicherheit und die zielgruppenspezifische Individualisierung der staatlichen Eingriffe allein nicht in der Lage sind, die Wohlfahrt der Bürger zu gewährleisten. Denn mit fortschreitender Differenzierung und Verselbständigung der durch die Selbstbeschrănkung des Staates freigesetzten und sich zunehmend organisierenden Handlungszusammenhänge entstehen systemische Dynamiken, deren Folgen für andere Handlungszusammenhänge, aber auch für die Bevölkerung als ganze zu weder individuell noch gruppenspezifisch zurechenbaren Nachteilen führen kőnnen. Deshalb wird nunmehr dem Staat die steuernde Beeinflussung dieser systemischen Zusammenhänge angesonnen. ${ }^{4}$

Welche Konsequenzen diese Veränderungen in der Zuschreibung von Staatsaufgaben für das Staatsverständnis und das staatliche Handeln haben, ist bisher kaum erforscht und bildet in Teilen ein wichtiges Thema dieses Bandes. Zunächst ist in Betracht zu ziehen, daß mit dem Auftauchen neuer Diskurse über Staatsaufgaben die Erfüllung der bisherigen Staatsaufgaben keineswegs überflüssig wird. Eher wird man annehmen dürfen, daß das Auftauchen neuer Diskurse ein Zeichen dafür ist, daß wesentliche Postulate der älteren Diskurse in der Praxis der Staatstätigkeit ihren Niederschlag gefunden haben. Aber eben diese Praxis muß auch im Zuge der Neuorientierung weiterlaufen und generiert dabei ebenfalls fortgesetzte

4 Luhmann (1990: 29, 37 f.) unterscheidet drei 'Sozialmodeller der Artikulation doppelter Kontingenz: das Modell der Normen, das Modell der Knappheit und das Modell des Risikos. bezogen auf unsere Typisierung von Staatsdiskursen, läßt sich die Normenthematik der Rechtsstaatlichkeit, Steuerungsstaatlichkeit zuordnen. 
Anpassungserfordernisse, die jedoch überwiegend auf der Ebene einzelner Politikfelder und nicht derjenigen von Diskursen über Staatsaufgaben erörtert werden.

Offensichtlich besteht ein erhebliches Unbehagen über diese Entwicklung auf Seiten der juristischen Staatslehre. Der Staat wird - so scheint es - mit immer neuen Zumutungen überfordert, deren ansatzweise Erfüllung sich überdies rechtsstaatlicher Kontrolle weitgehend entzieht. Durch die neueren gesellschaftstheoretischen Entwicklungen erscheint nunmehr der Staat auch nicht mehr als Gegenüber von ,Gesellschaft, sondern als institutioneller Kern des politischen Teilsystems von Gesellschaft. Am konsequentesten wird dies in der Theorie von Niklas Luhmann zu Ende gedacht, wo ,Gesellschaft - durchaus in radikalisierender Nachfolge zum letztlich auf Aristoteles zurückgehenden Konzept der ssocietas civilis - als $\bowtie$ Begriff für die Einheit der Gesamtheit des Sozialen« (1984: 555) gesetzt wird, womit der Staat notwendigerweise darunterfallt. Staatstheoretiker befürchten - und manche Soziologen bestātigen sie durch ihre staatskritischen Formulierungen in dieser Befürchtung - daB damit die herausgehobene, hoheitliche Funktion des Staates gegenüber den übrigen Teilsystemen der Gesellschaft verloren gehen könne. In der Tat kennt die Luhmannsche Theorie (wie übrigens die angelsächsische Soziologie seit jeher) kein Über- und Unterordnungsverhältnis gesellschafticher Teilsysteme, sondern einen allenfalls historisch wandelbaren funktionalen Primat.

Aber dadurch wird die konstitutive Funktion des Staates für moderne, hochkomplexe Sozialzusammenhänge nicht aufgegeben. Denn als die spezifische, d.h. nicht durch Leistung anderer Teilsysteme substituierbare Leistung des politischen Teilsystems gilt die Kommunikation über und die Herstellung von kollektiv bindenden Entscheidungen, die nicht nur für den Staat, sondern für alle gesellschaftlichen Teilbereiche - als staatlich gesetztes Recht - gelten. Solches Recht ist aber und dessen war sich bereits die liberale Staatstheorie durchaus bewußt - konstitutiv für die Stabilisierung anderer emergenter Sozialordnungen, beispielsweise für die Marktwirtschaft, das Gesundheits- und Bildungswesen oder selbst die Familie. ${ }^{5}$ Und es gehört demzufolge auch zu den nicht substituierbaren staatlichen Aufgaben, verbindliches Recht zu schaffen, das Recht zu schützen, Recht zu sprechen und Rechtsverstöße zu ahnden, also die Funktionserfordernisse des Rechtssystems zu gewährleisten, das seinerseits wesentliche Prămissen für alle sozialen Handlungszusammenhänge beinhaltet. Bezogen auf die Ordnung der sozialen Beziehungen kommt somit dem Staat eine konstruktive Funktion zu, da nahezu alle weiteren Formen der Ordnungsbildung an staatlich gesetzte Rechtsnormen und organisatorische Möglichkeiten (z.B. die verschiedenen Formen juristischer Per-

5 Inwieweit die Organisation des ausdifferenzierten politischen Systems jedoch notwendigerweise den Charakter eines Einheitsstaates tragen muB, wie die Souverånitătstheorien sowie die absolutistischen und nationalstaatlichen Interpretationen postulieren, scheint heute erneut eine offene Frage, die hier nicht zu behandeln ist. 
sönlichkeit) anknüpfen. Diese klassische Staatsfunktion gewinnt unter dem Gesichtspunkt der neuen Steuerungsaufgaben (vgl. II.4) eine zusătzliche, spezifische
Qualität.

Die klassische Unterscheidung von offentlichem und privatem Recht geht davon aus, daß das Recht zur Selbstbindung des Staates einerseits und das Rahmenrecht zur Ordnung der Beziehungen zwischen den Privaten andererseits zwei getrennte und den Bereich der Rechtsordnung erschöpfende Sphären darstellen. Das Ungenügen dieser Vorstellung wird insbesondere in den Bereichen des Wirtschafts-, Arbeits- und Sozialrechts bereits sichtbar, wo sich öffentlich-rechtliche und privatrechtliche Normen in ihrer Wirkung durchdringen. In dem MaBe, wie der Staat die Aufgabe der Steuerung ganzer Gesellschaftsbereiche wie z.B. der Gesundheits- (vgl. Rosewitz/Webber 1990) oder der Technologiepolitik (vgl. Werle 1990 ) übernimmt, deren Leistungen nahezu ausschließlich durch nichtstaatliche Akteure und deren Beziehungen zustande kommen, wird die Unterscheidung von
offentlichem und privatem Recht vollends obsolet.

Das gilt jedoch nicht z.B. für die Befriedigung unmittelbarer Lebensbedürfnisse, für die Produktion kultureller Deutungsmuster oder für die Reproduktion der Bevölkerung, welche ebenso konstitutive Bedeutung für den Fortgang der Geschichte der Menschengattung haben. Hier finden sich nach wie vor weitgehend staatsfreie Räume, die auch mit guten Gründen von politischen Einflüssen freigehalten werden. Insofern als staatliche Ordnung jedoch für einen identifizierbaren Teil der Erdoberfläche und dessen Bewohner Grenzen aufrichtet und damit einen Raum gesteigerter Interdependenz und Schicksalsverbundenheit konstituiert, den kulturelle Deutungen als kollektive Identitäten (z.B. ) Frankreich') und funktionale Zusammenhänge symbolisch repräsentieren, wird sie zum Garanten eines Sozialzusammenhangs, der auch diese Leistungen umfaßt. ${ }^{6}$ Auf diesen staatlich konstituierten Sozialzusammenhang beziehen sich die Diskurse über Staatsaufgaben, wobei im Zuge der Ausdifferenzierung und Thematisierung immer neuer Staatsaufgaben zweierlei stärker ins Bewußtsein tritt: zum einen die Interdependenz der staatlichen und nicht-staatlichen Leistungen bzw. der notwendige Synergismus zwischen beiden, und zum anderen der Verlust der Autarkie, die Partialităt solcher staatlicher Vergesellschaftung im Rahmen der entstehenden ,Weltgesellschaft. Daß Staaten keine 'souveränen` Akteure mehr sind, ergibt sich somit nicht nur aus der Interdependenz mit den übrigen Teilsystemen, sondern auch aus der wachsenden internationalen, im Grenzfall globalen Interdependenz der Staaten und ihrer Bürger. Das Denkmodell des isouveränen Staates` bezog seine historische Plausibilität aus der pazifizierenden und intergrativen Leistung der frühneuzeitlichen Staatsentwicklung, wo die Aufrichtung und Verteidigung der Staatsgrenzen die

6 Für derartig răumlich partialisierte, aber zumindest heute nicht mehr autarke Sozialszusammenhânge ist bei Luhmann - dies sel der Klarheit halber hinzugefügt - der Gesellschaftsbegriff eben-
falls ausgeschlossen! (vgl. Luhmann 1975: $60 \mathrm{f}$.). 
gen und die von Norbert Elias hervorgehobene Verlängerung der Handlungsketten wurde. In dem Maße jedoch, als das nationalstaatliche Ordnungsniveau selbstverständlich geworden ist, haben sich die Handlungsketten weiter verlängert und die Austauschbeziehungen über Staatsgrenzen hinweg in vielerlei Hinsicht so sehr intensiviert, daß Staatsgrenzen zunehmend keine Interdependenzunterbrecher mehr darstellen. Damit verliert der Staat notwendigerweise seine Souveränität, er wird nun funktional zu einer Art Organisationszentrum für die durch ihn selbst konstituierten Kollektivitäten, die sich eben nur durch ihn überhaupt noch als abgrenzbare Einheiten verstehen.?

Eine dieser neuen Problemlage angemessene Staatstheorie müBte m.E. die Prämisse der Souveränităt explizit verlassen und von den Konsequenzen des unvermeidlichen Souverānitätsverlustes ausgehen. Staatsaufgaben sind in doppelter Hinsicht als Funktionen in einem staatsübergreifenden Zusammenhang aufzufassen, nämlich zum einen mit Bezug auf die Entwicklungsperspektiven der staatlich konstituierten, aber im politischen Teilsystem nicht aufgehenden Kollektivităt, und zum anderen mit Bezug auf die inter- und überstaatlichen Beziehungen, in die die Staaten nunmehr als Staaten, d.h. als politisch verfaßte Kollektivitäten verflochten sind.

Die 'neue Unsicherheit , welche heute als $\backslash$ Risikogesellschaft oder als Forderung einer Staatsaufgabe, Risikovorsorge ${ }^{\prime}$ thematisiert wird, ist in der Tat primär ein Problem der ineuen Unübersichtlichkeit، (Habermas 1985). Die alten Denkmodelle, z.B. der kausalen Determiniertheit von Wirklichkeit oder der staatlichen Souveränitāt, mit denen der frühneuzeitliche Mensch sich seiner Position als "maitre et possesseur de la nature $z u$ vergewissern suchte, sind immer offenkundiger zum Scheitern verurteilt. Dennoch ist der Raum des in seinen Folgen Überschaubaren und daher grundsätzlich durch Entscheidungen Beeinflußbaren infolge der faktischen Verlängerung der Handlungsketten und ihrer immer komplexeren Repräsentation durch wissenschaftliche Erkenntnisse enorm gewachsen. Nicht die Gefährdungen des Menschen haben zugenommen (ein Blick auf die weltweit steigende Lebenserwartung genügt, um die Unsinnigkeit dieser Diagnose zu erkennen), sondern die Risiken, d.h. die Zurechenbarkeit möglicher Folgen auf Entscheidungen (vgl. Luhmann 1990: $34 \mathrm{ff}$.). Insofern als der Staat nach wie vor das akzeptierte Organisationsmodell zur Herstellung kollektiv bindender Entscheidungen darstellt, kommt ihm in dieser Situation eines zunehmenden Abhängigkeitsbewußtseins von einer unkontrollierbaren Zukunft bei gleichzeitig wachsenden Bewußtsein der Beeinflußbarkeit von Handlungszusammenhängen notwendigerweise eine nicht nur politische, sondern auch kulturelle, d.h. bedeutungsmäßige, Schlüsselfunktion zu: Der Glaube an die notwendigerweise wohltätige Wirksamkeit der sunsichtbaren Hand ist ebenso verblaßt wie der Glaube an die

7 Das gilt dann nicht nur für Nationalstaaten, sondern auch (schon seit langem) für Gliedstaaten im förderativen Staat, die bemerkenswerterweise mit der Abschwächung der nationalstaatlichen Souveränität wieder stärkere politische Bedeutung zu gewinnen scheinen. 
Allmacht eines starken Stantes. Die kollektiven Erwartungen mulssen bescheidener, aber auch differenzierter werden und die Koexistenz unterschiedlicher Steuerungsmodi menschlicher Handlungen sowie die in ihnen enthaltenen Lernmoglichkeiten genauer in den Blick nehmen (vgl. Kaufmann 1991). Eben hierfor ist Staatstheorie notwendig, d.h. die Entwicklung wirklichkeitsangemessenerer Vorstellungen vom Staat und seiner Wirkungsweise unter den Bedingungen entfalteter Modernităt und weltweiter Interdependenz. Dabei geht es jedoch nicht nur um beschreibende und erklärende Denkmodelle, sondern auch um die Entwicklung von normativen Kriterien, um die Zweckmåßigkeit politischer Forderungen beurteilen zu können. Die alten ordnungspolitischen Vorstellungen von zwei sich ausschlie. Benden Koordinationsmechanismen *Markt und Plan* b2w. wierarchische Steuerung* können den neuen Entwicklungen in keiner Weise mehr gerecht werden. Es geht vielmehr darum, Vorstellungen über das produktive Zusammenwirken von staatlichen und anderen, gesellschaftlichen Akteuren zu entwickeln und institutionelle Regelungen vorzuschlagen, um dessen Wahrscheinlichkeit zu erhöhen.

\section{Literaturverzeichnis}

Alber, Jens, 1982: Vom Armenhaus zum Wohlfahrtsstaat: Analysen zur Entwicklung der

Sozjalversicherung in Westeuropa. Frankfurt a.M.: Campus. Ashford, Douglas E., 1986: The Emergence of the Welfare States. Oxford: Blackwell. Beck, Ulrich, 1986: Risikogesellschaft. Frankfurt a.M.: Suhrkamp.

Berman, Harold J., 1983: Law and Revolution. The Formation of the Western Legal Tradition. Cambridge, Mass.: Harvard University Press. (deutsch: Frankfurt a.M.: Suhrkamp.
1991).

Berger, Peter/Thomas Luckmann, 1969: Die

keit. Eine Theorie der Wissenssoziologie. Fesellschaftliche Konstruktion der Wirklich-

Handwörterbuch der Wirtsc. „Marktwirtschaft, sozialex. In: Willi Albers u.a. (Hrsg.), Böckenforde, Ernst-Wolfgang, 1976a: Dichaft, Bd. 5. Stuttgart: Fischer.

Gesellschaft im demokratischen Gesellschaft. Darmstadt: Wissenschalstaat der Gegenwart. In: ders. (Hrsg.), Staat und Böckenförde, Ernst-Wolfgang, 1976b: Grutliche Buchgesellschaft, 395-431.

In: ders., Staat, Gesellschaft, Freiheit. Studiechtstheorie und Grundrechtsinterpretation. recht. Frankfurt a.M.: Suhrkamp, 221-252.

Böckenförde, Ernst-Wolfgang, 1976c: Lorenz von Stein als Theoretiker der Bewegung von Staat und Gesellschaft zum Sozialstaat. In: ders. (Hrsg.), Staat und Gesellschaft. Darmstadt: Wissenschaftliche Buchgesellschaft, 131-171.

Böckenförde, Ernst-Wolfgang. 1976d: Die politische Funktion wirtschaftlich-sozialer Verbände und Interessentrăger in der sozialstaatlichen Demokratie. In: Der Staat 15,
457-483. 
Bornschier, Volker, 1980: Multinationale Konzerne, Wirtschaftspolitik und nationale Entwicklung im Weltsystem. Frankfurt a.M.; Campus.

Bornschier, Volker/Peter Lengyel (Hrsg.) 1990: World Society Studies, Bd. 1. Frankfurt a.M.: Campus.

Conze, Werner, 1984: Stichw. »Sicherheit, Schutz.. In: Otto Brunner u.a. (Hrsg.), Geschichtliche Grundbegriffe, Historisches Lexikon zur politisch-sozialen Sprache in Deutschland, Bd. S. Stuttgart: Klett-Cotta, 831-862.

Dahme, Heinz-Jürgen/Dieter Grunow 1983: Persuasive Programme als Steuerungsinstrument des Wohlfahrtsstaates. Bielefeld: Kleine.

Delumeau, Jean, 1985: Angst im Abendland. Die Geschichte kollektiver Angste im Europa des 14. bis 18. Jahrhunderts. 2 Bde. Reinbek: Rowohlt.

Dyson, Kenneth, 1980: The State Tradition in Western Europe. Oxford: Robertson.

Edelman, Murray, 1976: Politik als Ritual. Die symbolische Funktion staatlicher Institutionen und politischen Handelns. Frankfurt a.M.: Campus.

Elias, Norbert, 1976: Über den Prozeß der Zivilisation. 2 Bde. Frankfurt a.M.: Suhrkamp.

Evers, Adalbert, 1989: Risiko und Individualisierung. In: Kommune, Nr. 6, 33-48.

Flora, Peter (Hrsg.), 1986 ff.: Growth to Limits - The Western European Welfare States since World War II. 5 Bde. Berlin: de Gruyter.

Forsthoff, Ernst (Hrsg.), 1968: Rechtsstaatlichkeit und Sozialstaatlichkeit. Darmstadt: Wissenschaftliche Buchgesellschaft.

Glagow, Manfred (Hrsg.), 1984: Gesellschaftssteuerung zwischen Korporatismus und Subsidiarităt. Bielefeld: AJZ.

Grimm, Dieter, 1986: Verfassungsrechtliche Anmerkungen zum Thema Prävention. In: Kritische Vierteljahresschrift für Gesetzgebung und Rechtswissenschaft 1, 38-54.

Grimm, Dieter, 1987a: Die sozialgeschichtliche und verfassungsrechtliche Entwicklung zum Sozialstaat. In: ders., Recht und Staat der bürgerlichen Gesellschaft. Frankfurt a.M.: Suhrkamp, 138-161.

Grimm, Dieter, 1987b: Zur politischen Funktion der Trennung von öffentlichem und privatem Recht in Deutschland. In: ders., Recht und Staat der bürgerlichen Gesellschaft. Frankfurt a.M.: Suhrkamp, 84-103.

Habermas, Jürgen, 1985: Die neue Unübersichtlichkeit. Frankfurt a.M.: Suhrkamp.

Häberle, Peter, 1972: Grundrechte im Leistungsstaat. In: Verhandlungen der Vereinigung deutscher Staatsrechtslehrer 30, 43-131.

Hegel, Georg Wilhelm Friedrich, 1968 (1821): Grundlinien der Philosophie des Rechts oder Naturrecht und Staatswissenschaften im Grundrisse. Frankfurt a.M.: Fischer.

Heimann, Eduard, 1980 (1929): Soziale Theorie des Kapitalismus - Theorie der Sozialpolitik. Frankfurt a.M.: Suhrkamp.

Heintz, Peter, 1982: Die Weltgesellschaft im Spiegel von Ereignissen. Diessenhofen: Ruegger.

Heller, Hermann, 1970: Staatslehre, 4. Aufl. Leiden: Sijthoff.

Hirschman, Albert O., 1980: Leidenschaften und Interessen - Politische Begründungen des Kapitalismus vor seinem Sieg. Frankfurt a.M.: Suhrkamp.

Hood, Christopher, 1986: The Hidden Public Sector: The 'Quangocratization ' of the World?

In: Franz-Xaver Kaufmann u.a. (Hrsg.), Guidance, Control, and Evaluation in the Public Sector. Berlin: de Gruyter, 183-207.

Humboldt, Wilhelm v., 1982 (1792): Ideen zu einem Versuch, die Grenzen der Wirksamkeit des Staats zu bestimmen. Stuttgart: Reclam. 
Jellinek, Georg, 1966 (1900): Allgemeine Staatslehre, Neudruck der 3. Aufl. Bad Homburg v.d.H.: Gehlen.

Kant, Immanuel, 1968 (1793): Über den Gemeinspruch: Das mag in der Theorie richtig sein, taugt aber nicht für die Praxis. In: Werke in 10 Bänden, hrsg. v. Wilhelm Weischedel, Bd. 9. 2. Aufl. Darmstadt: Wissenschaftliche Buchgesellschaft, 127-172.

Kaufmann, Franz-Xaver, 1973: Sicherheit als soziologisches und sozialpolitisches Problem, 2. Aufl. Stuttgart: Enke.

Kaufmann, Franz-Xaver, 1982: Elemente einer soziologischen Theorie sozialpolitischer Intervention. In: ders. (Hrsg.), Staatliche Sozialpolitik und Familie. München: Oldenbourg, 49-86.

Kaufmann, Franz-Xaver, 1986: Nationale Traditionen der Sozialpolitik und europāische Integration. In: Lothar Albertin (Hrsg.), Probleme und Perspektiven europäischer Einigung. Düsseldorf: Landeszentrale für politische Bildung NW, 69-82.

Kaufmann, Franz-Xaver/Giandomenico Majone/Vincent Ostrom (Hrsg.), 1986: Guidance, Control, and Evaluation in the Public Sector. The Bielefeld Interdisciplinary Project. Berlin: de Gruyter.

Kaufmann, Franz-Xaver, 1991: The Relationship between Guidance, Control, and Evalution. In: ders. (Hrsg.), The Public Sector. Challenge for Coordination and Learning. Berlin: de Gruyter, 213-234.

Kittsteiner, Heinz-Dieter, 1984: Ethik und Teleologie: Das Problem der unsichtbaren Hand bei Adam Smith. In: Franz-Xaver Kaufmann/Hans-Günter Krüsselberg (Hrsg.), Markt, Staat und Solidarität bei Adam Smith. Frankfurt a.M.: Campus, 41-73.

Küng, Emil, 1956: Stichw. "Interventionismus*. In: Handwörterbuch der Sozialwissenschaften, Bd. S. Stuttgart: Fischer, 321-329.

Luhmann, Niklas, 1968: Zweckbegriff und Systemrationalität. Über die Funktion von Zwecken in sozialen Systemen. Tübingen: Mohr.

Luhmann, Niklas, 1975: Die Weltgesellschaft. In: Soziologische Aufklärung, Bd. 2. Opladen: Westdeutscher Verlag, 51-71.

Luhmann, Niklas, 1981: Politische Theorie im Wohlfahrtsstaat. München: Olzog.

Luhmann, Niklas, 1990: Risiko und Gefahr. Aula-Vortrăge der Hochschule St. Gallen Nr. 48.

Mayntz, Renate (Hrsg.), 1983: Implementation politischer Programme: Ansătze zur Theoriebildung. Opladen: Westdeutscher Verlag.

Mayntz, Renate/Bernd Rosewitz/Uwe Schimank/Rudolf Stichweh, 1988: Differenzierung und Verselbståndigung. Zur Entwicklung gesellschaftlicher Teilsysteme. Frankfurt: Campus.

Meadows, Dennis u.a., 1972: Die Grenzen des Wachstums. Bericht des Club of Rome zur Lage der Menschheit. Stuttgart: Deutsche Verlagsanstalt.

Destreich, Gerhard, 1969: Strukturprobleme des europäischen Absolutismus. In: ders., Geist und Gestalt des frùhmodernen Staates. Ausgewählte Aufsătze. Berlin: Duncker \&
Humblot.

Parsons, Talcott, 1972: Das System moderner Gesellschaften. München: Juventa.

Preu, Peter, 1983: Polizeibegriff und Staatszwecklehre. Göttingen: Schwartz.

Riner, Gerhard A., 1988: Der Übergang zum Interventions- und Wohlfahrtsstaat und des. sen Auswirkungen auf Parteien und Parlamente im deutschen Kaiserreich. In: Wilhelm Treue (Hrsg.). Geschichte als Aufgabe. Festschrift für Otto Büsch. Berlin: Colloquium
Verlag, 437-459. 
Rosewitz, Bernd/Douglas Webber, 1990: Reformversuche und Reformblockaden im deutschen Gesundheitswesen. Frankfurt: Campus.

Saladin, Peter/Christoph Andreas Zenger, 1988: Recht künftiger Generationen. Basel: Helbing \& Lichtenhahn.

Scholz, Franz, 1955: Die Rechtssicherheit. Berlin: de Gruyter.

Schuppert, Gunnar $F$; 1981: Die Erfüllung öffentlicher Aufgaben durch verselbständigte Verwaltungseinheiten. Göttingen: Schwartz.

Teubner, Gunther, 1989: Recht als autoproietisches System. Frankfurt a.M.: Suhrkamp.

Teubner, Gunther/Helmut Willke, 1984: Kontext und Autonomie: Gesellschaftliche Selbststeuerung durch reflexives Recht. In: Zeitschrift für Rechtssoziologie 5, 4-35.

Werle, Raymund, 1990: Telekommunikation in der Bundesrepublik. Frankfurt a.M.: Campus.

Willke, Helmut, 1983: Entzauberung des Staates. Überlegungen zu einer sozietalen Steuerungstheorie. Königstein/Ts.; Athenäum.

Willke, Helmut, 1988: Staatliche Steuerung als Kontextsteuerung. Am Beispiel Eureka. In: Kritische Vierteljahresschrift für Gesetzgebung und Rechtswissenschaft 3, 214-229.

Willke, Helmut, 1991: Le droit comme instrument de guidage neomercantiliste de l'état. In: Charles-Albert Morand (Hrsg.), L'état propulsif. Contribution a l'étude des instruments d'action de l'etat. Paris: Poblisud, 95-106.

Zacher, Hans F., 1980: Sozialpolitik und Verfassung im ersten Jahrzehnt der Bundesrepublik Deutschland. Berlin: Schweitzer. 